\title{
Anaesthesia and Intensive Care Residents' Perception of Simulation Training in Four Romanian Centres
}

\author{
Horațiu N Vasian ${ }^{*}$, Simona C Mărgărit¹ ${ }^{1}$ Ioana Grigoraș², Leonard Azamfirei³ ${ }^{3}$ Dan \\ Corneci ${ }^{4}$, Daniela Ionescu ${ }^{1,5}$ \\ 1 Iuliu Hațieganu University of Medicine and Pharmacy, $1^{\text {st }}$ Department of Anaesthesia and Intensive Care, Cluj- \\ Napoca, Romania \\ 2 Department of Anaesthesiology and Intensive Care, Gr. T. Popa University of Medicine and Pharmacy, Iași, Romania \\ 3 Department of Intensive Care, University of Medicine and Pharmacy, Târgu-Mureș, Romania \\ 4 Department of Anaesthesiology and Intensive Care, Carol Davila University of Medicine and Pharmacy, Bucharest, \\ Romania \\ ${ }^{5}$ Outcome Research Consortium, Cleveland, Ohio, USA
}

\begin{abstract}
Introduction: Simulation training offers an opportunity to educate anaesthesia and intensive care (AIC) residents safely. At present, it is not yet a mandatory part of residency curriculum.

The aim of the study was to evaluate the residents' perception of the inclusion of simulation-based training in the Romanian AIC residency program.

Material and methods: Romanian AICs in anaesthesia and intensive care from four training centres completed a twenty-question survey regarding their views on simulation during their residency training. Residents were divided into junior, in the first three years, or senior groups, in the last two years of residency training. The questionnaire included Likert-scale multiple-choice, open-ended, and "yes" or "no" questions regarding simulator learning, the frequency of simulation sessions, and the value of the simulation sessions in improving practice, skills or teamwork. The open-ended questions, asked which were the respondents' preferred topics to be included in simulation sessions.

Results: Fifty-six percent of residents completed and returned the questionnaire. Ninety-eight percent of them considered simulation-learning useful once a month or every three months especially in the first two years of training. All residents thought simulation sessions would improve their skills, communication abilities, and teamwork. Senior residents paid more attention to clinical scenarios $(p=0.007)$, haemodynamic monitoring $(p=0.017)$ and mechanical ventilation $(p=0.004)$ as compared to juniors. All residents considered difficult airway management, and cardiac life support to be very important issues to be included in simulation sessions.
\end{abstract}

Conclusion: The survey demonstrated that simulation-based training should play a greater role and eventually became compulsory in training program in AIC academic centres.

Keywords: residents' perception, anaeshtesia and intensive care, training, survey, simulation

Received: 18 December 2016 / Accepted: 5 January 2017

\section{INTRODUCTION}

Simulation technology is increasingly used in medical education to increase patient safety. Medical simulation is a tool used to mimic a real situation not only for educational purpose but also for evaluation of professional competence [1].
During residency, simulation training adds value to training programs by offering residents the opportunity to acquire skills and practice scenarios in a safe and realistic learning environment without jeopardising patient safety [2,3]. It allows skills to be practised and to be repeated at any time during training progress 
as well as postgraduate courses. Unlike traditional lectures, medical simulation provides a means to assess knowledge, skills, and communication capabilities and integrate these into a clinical context $[4,5]$.

Simulation-based medical education has grown significantly worldwide and is now an important part of many anaesthesia training programs [6-9]. However, residents' perception of simulation programs has been evaluated in only a few reported studies $[4,10,11]$.

The aim of the present study was to assess Romanian anaesthesia and intensive care residents' perception of simulation training as a potential compulsory part of their medical education taking into consideration that such programs have been only recently introduced in some of academic training centres. This is the first study to evaluate Romanians anaesthesia and intensive care residents' perception on the simulation training.

\section{- MATERIALS AND METHODS}

Program directors from four Romanian anaesthesiology training centres, Cluj-Napoca, Târgu-Mureș, București, and Iași were contacted and invited to take part in the survey. The invitation included a web link to the online survey, was e-mailed to program coordinators who distributed the documents to all residents on their departmental e-mailing list.

All Romanian anaesthesiology and intensive care residents from the $1^{\text {st }}$ to $5^{\text {th }}$ years of training who were listed on each of the four university's departmental emailing lists were included in the study and invited to complete the questionnaire. There were no exclusion criteria.

Residents in their first to third years of residency training were considered "junior residents", and residents in their final two years of residency training were considered "senior residents".

The survey was electronically distributed on March 2015 to all residents and was made available for completing on line for three month, from March $1^{\text {st }}$ to May $31^{\text {st }}, 2015$. Three reminder e-mails were sent to program coordinators from all 4 centres during the $4^{\text {th }}, 8^{\text {th }}$ and $11^{\text {th }}$ weeks of online survey availability.

\section{Questionnaire development}

A twenty-question survey was developed to collect information from residents, regarding the perception of the role of simulation in their residency training. The residents were invited to complete a fifteen-minute voluntary, anonymous survey using the Google Forms online platform.

The questionnaire was designed based on a literature search and author's personal experience. The literature search was limited to human and English language articles. MEDLINE (1964 to March 2015) and EMBASE (1978-March 2015) were searched using terms "medical simulation", "anaesthesia simulation training", "high-fidelity simulation", and permutations thereof.

Prior sending to respondents, the questionnaire was analysed by all four program coordinators.

The survey was not piloted on anaesthesia residents before sending the questionnaire.

The questionnaire included Likert-scale multiplechoice responses, open-ended questions and "yes" or "no" questions.

For multiple-choice questions, the respondents were asked to indicate their agreement with each statement ranging from strongly agree to disagree strongly. Each response received a numerical value: strongly agree (1), agree (2), disagree (3), strongly disagree (4) and don't know (5).

Multiple-choice questions included issues such as:

- usefulness of simulator learning

- most appropriate time for simulation during the 5th year training

- the option of the inclusion of simulation as a mandatory part of residency curriculum

- the frequency of the simulation sessions during an academic year

- the value of the simulation sessions in improving practice skills

- communication capabilities and teamwork.

For the open-ended questions, the respondents had to give their preferred topics to be included in future simulation sessions.

The questionnaire included demographics, previous experience in the medical volunteering field, medical experience abroad and driving license.

The questionnaire can be found in Appendix 1 (available as Electronic Supplementary Material).

\section{Statistics}

Statistical analysis was performed using MedCalc Statistical Software 16.2.0 (MedCalc Software bvba, Ostend, Belgium). Quantitative variables, such as the 
resident's age, are presented as medians (interquartile range). Qualitative variables were presented as frequency and percentage. Comparisons between the frequencies of two or more variables between junior and senior resident groups were made using chi-square test or Fisher $\mathrm{t}$ exact test, accordingly. $\mathrm{P}$ values are compared with the "set" a value.

For certain multiple choice questions, raw data were presented as percentages of respondents "agreeing", "disagreeing 'or "don't know" with the statements, according to the Likert scale used.

Open-ended responses were divided into categories from which a "Top 5" most frequent categories of responses in both groups of residents, junior and senior, was developed.

\section{RESULTS}

\section{Demographics}

The questionnaire was distributed to 215 residents.

- 44 residents from University of Medicine and Pharmacy "Iuliu Hațieganu" Cluj-Napoca, Regional Institute of Gastroenterology - Hepatology "Prof Dr O Fodor" Cluj-Napoca;

- 49 residents from University of Medicine and Pharmacy Târgu-Mureș;

- 92 residents from University of Medicine and Pharmacy Iași

- 30 residents from University of Medicine and Pharmacy "Carol Davila", Elias Emergency University Hospital, Bucharest.

One hundred and twenty-two residents completed the questionnaire (56.7\%).

None of the residents enrolled in the study had previously participated in simulation sessions as a part of residency curriculum.

The response rate from all centres was presented in Figure 1. Responses by year of training and gender are shown in Figure 2.

Responders, age expressed as median, and the 25th and 75th percentile was 28 (26-30). One-third of the responders were males, and two-thirds were females.

\section{Resident's perception on simulation training}

Ninety-eight percent of respondents answered "yes" to the question "do you consider simulator learning useful during residency training?" Junior and senior residents
(45.9\%) believed the simulation sessions are most useful during the first three years of residency training.

The simulation sessions were considered useful only during the first year of training by $35.2 \%$ of all responders (Figure 3).

Regarding the frequency of the simulation sessions during an academic year, $68 \%$ of responders considered the optimal interval for simulation session was once a month or every three months (Figure 4). Fortyeight percent of senior residents felt once a month the most appropriate time for simulation session compared to junior residents.

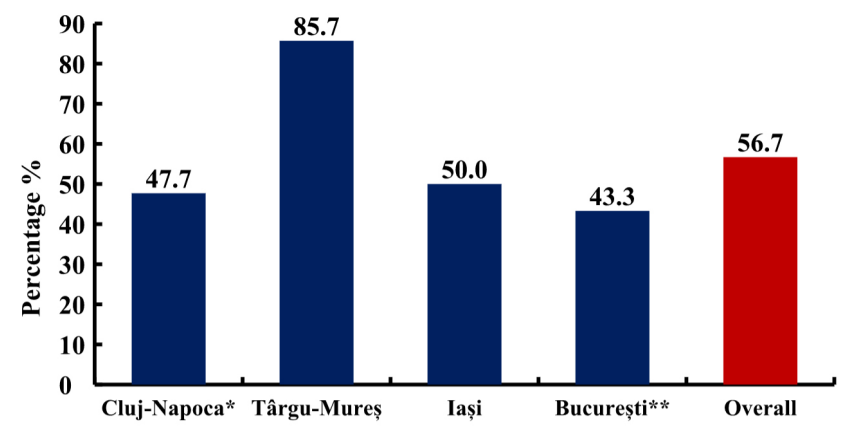

* Regional Institute of Gastroenterology - Hepatology "Prof Dr O Fodor" Cluj-Napoca, ** Elias Emergency University Hospital Bucharest

Fig. 1. Residents' response rate from all training centres

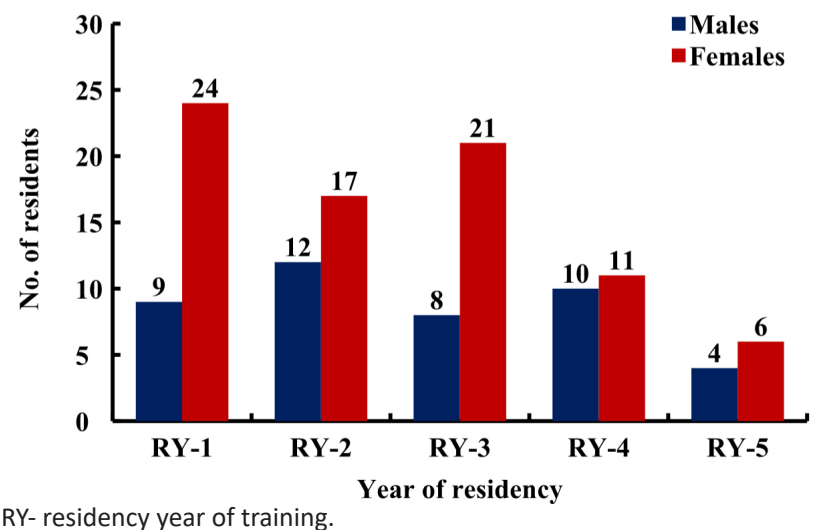

Fig. 2. Residents' response rate by residency training level and gender

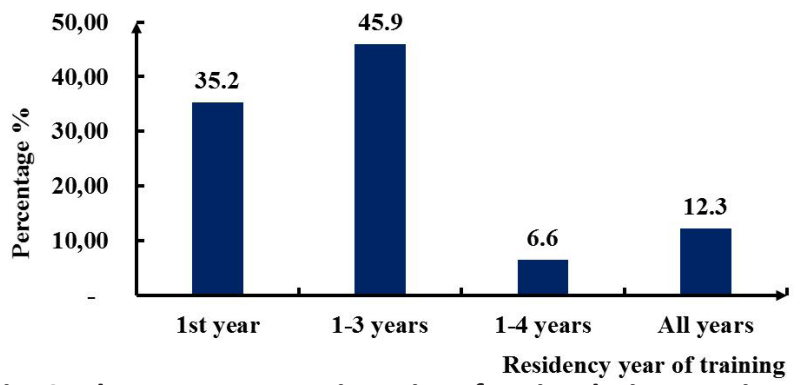

Fig. 3. The most appropriate time for simulation sessions during residency training programme 
Eighty-seven percent of all respondents answered positively to the question "should simulation education courses be mandatory in the anaesthesia and intensive care core curriculum?" Only $9 \%$ of all responders disagreed with the statement.

Ninety-eight percent of responders indicated that simulator training would improve their practice of safe anaesthesia, skills, communication abilities, teamwork, and consolidate their theoretical knowledge.

Regarding the preferred method for improving skills, abilities and theoretical knowledge, $60 \%$ of responders considered simulation session to be the preferred method compared to information provided by the internet, films or videos. (Figure 5).

The proposed simulation scenarios of primary interest to the respondents are given in Figure 6.

The "top five" topics ranked by junior residents included:

- difficult airway management (20.9\%)

- basic and advanced cardiac life support (13.2\%)

- general and regional anaesthesia techniques $(12.1 \%)$

- clinical scenarios (6.6\%); (5) hemodynamic monitoring (4.4\%)

- For senior residents, the top 5 topics were:

- difficult airway management (35.5\%)

- general and regional anaesthesia techniques (25.8\%)

- basic and advanced cardiac life support (25.8\%)

- clinical scenarios (25.8\%); (5) hemodynamic monitoring (19.4\%)

Senior residents were more interested in complex topics such as mechanical ventilation $(\mathrm{p}=0.004)$, haemodynamic monitoring $(\mathrm{p}=0.017)$ and clinical scenarios $(\mathrm{p}=0.007)$ (Figure 6).

\section{DISCUSSIONS}

Traditionally, anaesthesia and intensive care residency training consisted of oral lectures and presentations, case presentations and performing procedures on patients under supervision. However, simulation presents a more efficient training tool for acquiring the necessary skills to perform invasive procedures and nontechnical skills; such as team working, situation awareness, decision making, and task management [4,6]. Simulation allows educators to create training exercises

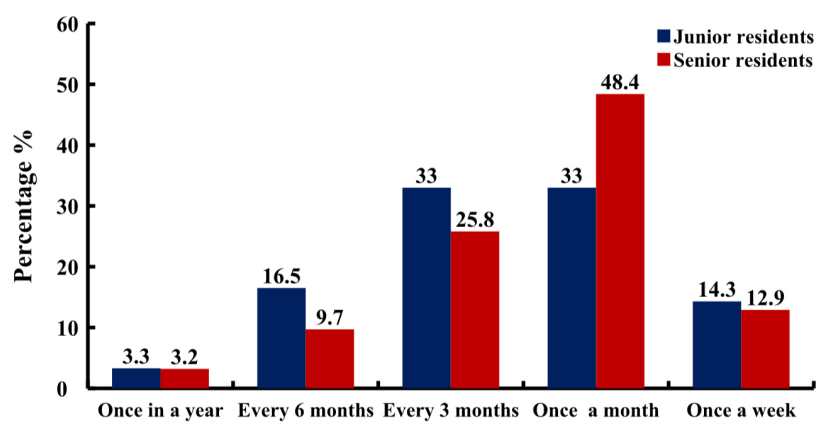

Fig. 4. Optimal schedule for simulation sessions during an academic year as suggested by respondents

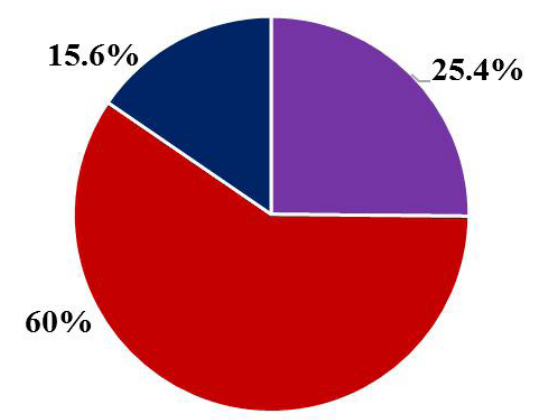

- Internet - Simulation session - Don't know

Fig. 5. Learning method preferred for improving skills, abilities, knowledge

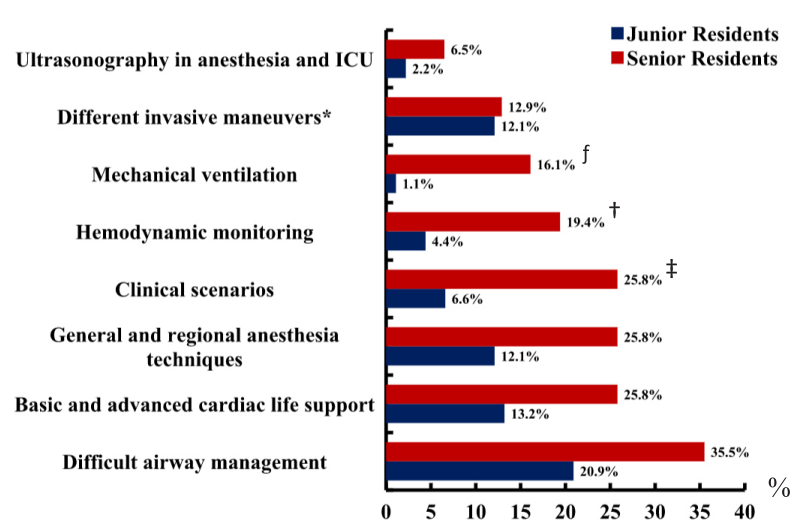

* Different invasive manoeuvres included peripheral and central venous catheterization, thoracentesis, and other basic manoeuvres in anaesthesia and intensive care. ${ }^{f} p=0.004,{ }^{\dagger} p=0.017,{ }^{\ddagger} p=0.007$

Fig. 6. Description of the preferred topics for simulation session. Results were expressed as a percentage of responders for every topic reported to a total number of responders in each group (junior or senior)

in an environment that reduces patient safety concerns and permits repetitive practice $[1,4,6]$.

The major barriers in simulation training worldwide is a lack of funding (50\%) and lack of faculty resources $(27 \%)$, even in developed or developing countries. 
The benefits are experimental learning (66\%) and the management of critical or rare events (37\%) [2]. Anaesthesiology residents trained on simulators were found to responded more quickly and performed procedure more efficiently [12].

In the present survey, the perception of residents regarding the importance of simulation-based learning during their five years training was evaluated, even though they had no educational experience with highfidelity simulation. From the academic centres contacted for the survey, two out of the four, Cluj-Napoca and Târgu-Mureș respectively, have included practical skills and medical simulation centres for undergraduate and postgraduate students and resident medical staff.-

The residents' responses were in favour of this learning method. They considered simulator training as a tool to improve their practice, skills, communication abilities, teamwork, consolidate their theoretical knowledge.

In accord with the current data, Canadian anaesthesiology residents considered simulation-based education valuable for enhancing their capacity to deal with emergency situations [10], though they found training with high-fidelity simulation as an anxiety provoking experience.

Cardiac life support and airway management were considered the top two preferred topics for simulation sessions by both Romanian and Canadian junior residents, though malignant hyperthermia and obstetrical emergencies were the main two subjects preferred by senior Canadian anaesthesia residents [10].

Unlike Canadian residents, Romanian senior residents preferred simulation session on haemodynamic monitoring, clinical scenarios, and mechanical ventilation to a larger extent than junior residents. A possible explanation for this result may be the differences in infrastructure among different ICUs and operating rooms and differences in methods of training between centres.

Care should be taken in generalising the results of the present study as the four centres may not be entirely representative of Romanian anaesthesia and intensive care training centres for residents, though there are only two other such centres in Romania.

The overall response rate was $56.7 \%$, despite three attempts to encourage the non-responders to complete the questionnaire. However, the overall response rate is similar to other studies [13] and higher than a previous published international survey [10].

In conclusion, the study found that most participating residents considered it useful to have simulationbased training. Based on these findings, program coordinators should consider introducing simulation as mandatory during residency in Romania.

\section{CONFLICT OF INTEREST}

None declared.

\section{ACKNOWLEDGEMENT}

All authors participated equally in carrying out the study and drafting of the article.

\section{REFERENCES}

1. Lammers RL: Simulation: the new teaching tool. Ann Emerg Med. 2007;49:505-507.

2. Morgan PJ, Cleave-Hogg D: A worldwide survey of the use of simulation in anaesthesia. Can J Anaesth. 2002;49(7):659-62.

3. Okuda Y, Bryson EO, DeMaria S Jr et al.: The utility of simulation in medical education: what is the evidence? Mt Sinai J Med. 2009;76:330-3.

4. Meguerdichian DA, Heiner JD, Younggren BN: Emergency medicine simulation: a resident's perspective. Ann Emerg Med. 2012;60(1):121-6.

5. Steadman RH, Coates WC, Huang YM et al.: Simulationbased training is superior to problem-based learning for the acquisition of critical assessment and management skills. Crit Care Med. 2006;34:151-7.

6. Chang $\mathrm{CH}$ : Medical simulation is needed in anaesthesia training to achieve patient's safety. Korean J Anesthesiol. 2013;64(3):204-11.

7. Beydon L, Dureuil B, Nathan N, Piriou V, Steib A, Collège français des anesthésistes réanimateurs (Cfar): High fidelity simulation in anaesthesia and intensive care: context and opinion of performing centres-a survey by the French College of Anesthesiologists and Intensivists. Ann Fr Anesth Reanim. 2010;29(11):782-6.

8. Lassalle V, Berton J, Bouhours G, Péres M, Bossard G, Granry JC: Medical paediatric simulation: a European survey. Ann $\mathrm{Fr}$ Anesth Reanim. 2009;28(7-8):628-33.

9. Lorello GR, Cook DA, Johnson RL, Brydges R: Simulation-based training in anesthesiology: a systematic review and metaanalysis. Br J Anaesth. 2014;112(2):231-45.

10. Price JW, Price JR, Pratt DD, Collins JB, McDonald J: High-fidelity 
Available online at: www.jccm.ro

simulation in anesthesiology training: a survey of Canadian anesthesiology residents' simulator experience. Can J Anaesth. 2010;57(2):134-42.

11. Blum RH, Boulet JR, Cooper JB, Muret-Wagstaff SL; Harvard Assessment of Anesthesia Resident Performance Research Group: Simulation-based assessment to identify critical gaps in safe anaesthesia resident performance. Anesthesiology.
The Journal of Critical Care Medicine 2017;3(1) • 23 2014;120(1):129-4.

12. Chopra V, Gesink BJ, de Jong J, Bovill JG, Spierdijk J, Brand R: Does training on an anaesthesia simulator lead to improvement in performance? Br J Anaesth. 1994;73:293-7.

13. Savoldelli GL, Naik VN, Hamstra SJ, Morgan PJ: Barriers to use of simulation-based education. Can J Anaesth. 2005;52 (9):94450 . 